\title{
Optimal Power Allocation for Channel Estimation in MIMO-OFDM System with Per-Subcarrier Transmit Antenna Selection
}

\author{
Kaleeswaran RAJESWARI ${ }^{1}$, S. Jayaraman THIRUVENGADAM ${ }^{1,2}$ \\ ${ }^{1}$ Dept. of Electronics and Communication Engg., Thiagarajar College of Engineering, 625015 Madurai, TamilNadu, India \\ ${ }^{2}$ TIFAC CORE in Wireless Technologies, Thiagarajar College of Engineering, 625015 Madurai, TamilNadu, India \\ rajeswari@tce.edu, sjtece@tce.edu
}

\begin{abstract}
A novel hybrid channel estimator is proposed for multiple-input multiple-output orthogonal frequency-division multiplexing (MIMO-OFDM) system with per-subcarrier transmit antenna selection having optimal power allocation among subcarriers. In practice, antenna selection information is transmitted through a binary symmetric control channel with a crossover probability. Linear minimum mean-square error (LMMSE) technique is optimal technique for channel estimation in MIMO-OFDM system. Though LMMSE estimator performs well at low signal to noise ratio (SNR), in the presence of antenna-to-subcarrier-assignment error (ATSA), it introduces irreducible error at high SNR. We have proved that relaxed MMSE (RMMSE) estimator overcomes the performance degradation at high SNR. The proposed hybrid estimator combines the benefits of LMMSE at low SNR and RMMSE estimator at high SNR. The vector mean square error (MSE) expression is modified as scalar expression so that an optimal power allocation can be performed. The convex optimization problem is formulated and solved to allocate optimal power to subcarriers minimizing the MSE, subject to transmit sum power constraint. Further, an analytical expression for SNR threshold at which the hybrid estimator is to be switched from LMMSE to RMMSE is derived. The simulation results show that the proposed hybrid estimator gives robust performance, irrespective of ATSA error.
\end{abstract}

\section{Keywords}

MIMO-OFDM, per-subcarrier transmit antenna selection, antenna-to-subcarrier assignment, LMMSE, relaxed MMSE, hybrid estimator

\section{Introduction}

Orthogonal frequency division multiplexing (OFDM) is a popular method for high data rate wireless transmission [1]. Wireless standards such as digital audio broadcasting (DAB), digital video broadcasting-terrestrial (DVB-T), the IEEE 802.11a local area network (LAN) and the IEEE 802.16a metropolitan area network (MAN) have adopted OFDM technology. OFDM is also a potential candidate for fourth-generation (4G) mobile wireless systems. When OFDM is combined with multiple-input multiple-output (MIMO) system, it converts the frequency selective channel of the MIMO channel into a set of parallel frequencyflat channels. This decreases the MIMO receiver complexity [2]. Further, MIMO system provides spatial diversity by having spatially separated antennas [3]. Combination of OFDM and MIMO aims to increase the diversity gain and/or to enhance the system capacity [4]. Further, antenna selection in MIMO-OFDM systems provide considerable gains while only requiring a small amount of feedback to convey information to the receiver about the chosen transmit antennas [5-7]. The antenna to subcarrier assignment (ATSA) is signaled in a control channel from the transmitter to receiver.

In MIMO-OFDM systems, the antenna selection can be performed based on group-of-subcarriers or on a persubcarrier. In bulk selection, one or more antennas are chosen among the available antennas, based on signal to noise ratio or based on performance metrics such as capacity or bit error rate (BER), for transmission on all frequencies. This reduces channel state information (CSI) feedback requirement and number of radio frequency (RF) chains. The per-tone selection is capable of achieving a much lower BER as it exploits an additional degree of freedom that allows the antenna selection to differ across the utilized bandwidth [8], [9].

In MIMO-OFDM system with per tone transmit antenna selection, channel estimation is the most essential task in compensating distortion from channels. LMMSE based channel estimation technique performs well in rapid dispersive fading channels and it is proved to be robust against channel power delay profile [10-12]. In [13], LMMSE channel estimator for a system employing per tone selection is derived. The performance of LMMSE based channel estimator in MIMO-OFDM system is improved by combining with optimal power allocation [15-18]. Extending this to per tone antenna selection scheme is not simple as the autocorrelation matrix is noninvertible due to fluctuations in its rank. This paper pro- 
poses a LMMSE based channel estimation along with optimal power allocation scheme for MIMO-OFDM with per tone antenna selection system. An eigenvalue decomposition (EVD) of the autocorrelation matrix is employed to obtain a scalar MSE expression such that optimal power allocation scheme can be applied. A convex optimization framework is formulated to minimize the channel estimation MSE subject to sum power constraint. The improvement in the performance of LMMSE estimate with optimal power allocation is validated through MSE and BER analysis.

When LMMSE channel estimator is used in the system employing per tone selection with ATSA error, it results in intolerable performance at high SNR. This is due to error introduced in channel frequency correlation [12]. To overcome this problem in the system with equal power allocation to subcarriers, a hybrid estimator is proposed in [13]. This paper extends the design of hybrid estimator with optimal power allocation to subcarriers. Hybrid estimator requires less knowledge about the channel correlation and robust to ATSA error. It improves the performance of LMMSE estimator by switching to relaxed MMSE which is less complex and easy to implement. A closed form expression for the SNR threshold at which the estimator switches from LMMSE to relaxed MMSE is derived analytically.

The paper is organized as follows. System model with per subcarrier transmit antenna selection and maximal ratio combining at the receiver is presented in Sec. 2. The effect of incorrect antenna selection in the channel estimation is analyzed in Sec. 3. The proposed method of estimating CSI is described in Sec. 4. The performance of the proposed technique is analyzed by simulations in Sec. 5. Section 6 concludes the paper. In this paper, boldface letters are used to denote matrices and vectors. Superscript $H$ denotes Hermitian operations, $\otimes$ denotes the Kronecker product and $E[]$ denotes the expectation operation. $\operatorname{vec}(\mathbf{C})$ transforms a matrix $\mathbf{C}=\left[\mathbf{c}_{1}, \mathbf{c}_{2}, \ldots, \mathbf{c}_{n}\right]$ into a column vector $\left[\mathbf{c}_{1}^{T}, \mathbf{c}_{2}^{T}, \ldots, \mathbf{c}_{n}^{T}\right]$, where $\mathbf{c}_{i}$ is the $i^{\text {th }}$ column vector of $\mathbf{C}$.

\section{System Model}

Consider a MIMO-OFDM system with $n_{t}$ transmit antennas and $n_{r}$ receive antennas. It is assumed that the OFDM symbol has $N$ subcarriers. Let $\mathbf{g}(i, j)$ be a $L \times 1$ channel impulse response vector between the $j^{\text {th }}$ transmit antenna and the $i^{\text {th }}$ receive antenna. The corresponding channel frequency response (CFR) between the $j^{\text {th }}$ transmit antenna and the $i^{\text {th }}$ receive antenna at the $k^{\text {th }}$ subcarrier is given by

$$
f_{k}(i, j)=\sum_{l=0}^{L-1} g_{l}(i, j) e^{-j 2 \pi l k / N}, \quad k=0,1 \ldots N-1 .
$$

At each subcarrier, one of the $n_{t}$ transmit antennas is selected for transmission. The criterion for selecting the $j^{\text {th }}$ transmit antenna is given by

$$
\hat{j}_{k}=\underset{j \in\left[1,2 \ldots n_{t}\right]}{\arg \max }\left\{\sum_{i=1}^{n_{r}}\left|f_{k}(i, j)\right|^{2}\right\}, \quad k=0,1 \ldots N-1 .
$$

This ATSA information is signaled through a binary symmetric control channel to the receiver. The $N \times 1$ receive signal vector at the $i^{\text {th }}$ receive antenna is given by

$$
\mathbf{y}^{(i)}=\mathbf{X h}^{(i)}+\mathbf{w}^{(i)}
$$

where $\mathbf{X}$ is a $N \times N$ diagonal matrix, defined as $\mathbf{X}=\operatorname{diag}\left(x_{0}, x_{1}, \ldots, x_{N-1}\right)$ and $x_{k}=\sqrt{p_{k}} b_{k}$. The data on the $k^{\text {th }}$ subcarrier $b_{k}$ is assumed to be a random variable with zero mean and unit variance and $p_{k}$ is power of the $k^{\text {th }}$ subcarrier. $\mathbf{h}^{(i)}$ is a $N \times 1$ selected CFR vector at the $i^{\text {th }}$ receive antenna, defined as

$$
\mathbf{h}^{(i)}=\mathbf{S}_{o} \mathbf{f}^{(i)}
$$

where

$$
\mathbf{f}^{(i)}=\left[\mathbf{f}(i, 1)^{T} \mathbf{f}(i, 2)^{T} \ldots \mathbf{f}\left(i, n_{t}\right)^{T}\right]
$$

$\mathbf{f}(i, j)$ is the $N \times 1$ CFR vector between the $j^{\text {th }}$ transmit antenna and the $i^{\text {th }}$ receive antenna. $\mathbf{S}_{o}$ is a $N \times N n_{t}$ selection matrix given by

$$
\mathbf{S}_{o}=\left[\begin{array}{llll}
\operatorname{diag}\left(\mathbf{a}_{1}\right) & \operatorname{diag}\left(\mathbf{a}_{2}\right) & \ldots & \operatorname{diag}\left(\mathbf{a}_{n_{t}}\right)
\end{array}\right]
$$

where $\mathbf{a}_{j}$ is $N \times 1$ vector with its $k^{\text {th }}$ element being unity if the $j^{\text {th }}$ antenna is selected for the $k^{\text {th }}$ subcarrier transmission. $\mathbf{w}^{(i)}$ is the $N \times 1$ noise vector with mean zero and covariance matrix $\sigma_{w}^{2} \mathbf{I}_{N}$ of the $i^{\text {th }}$ receive antenna.

\section{Optimal Power Allocation} Algorithm

$\mathbf{R}_{\mathbf{h}}{ }^{(i)}$ is a Hermitian and positive definite matrix with the eigenvalue decomposition

$$
\mathbf{R}_{\mathbf{h}}^{(i)}=\mathbf{U}^{(i)} \boldsymbol{\Lambda}^{(i)}\left(\mathbf{U}^{(i)}\right)^{H}
$$

where $\mathbf{U}^{(i)}$ is a unitary matrix and $\Lambda^{(i)}$ is $N \times N$ diagonal matrix with non zero diagonal elements $\left[\lambda_{0}^{(i)}, \lambda_{1}^{(i)}, \ldots, \lambda_{r_{c}-1}^{(i)}\right]$. The channel CFR vector $\mathbf{h}^{(i)}$ is assumed to be Gaussian with zero mean and auto correlation matrix $\mathbf{R}_{\mathbf{h}}{ }^{(i)}$ of size $N \times N$. The LMMSE estimation of selected CFR vector $\mathbf{h}^{(i)}$ is given by [19]

$$
\hat{\mathbf{h}}^{(i)}=E\left[\mathbf{h}^{(i)}\left(\mathbf{y}^{(i)}\right)^{H}\right] E\left[\mathbf{y}^{(i)}\left(\mathbf{y}^{(i)}\right)^{H}\right]^{-1} \mathbf{y}^{(i)} .
$$


Substituting (3), the statistical average of $E\left[\mathbf{h}^{(i)}\left(\mathbf{y}^{(i)}\right)^{H}\right]$ and $E\left[\mathbf{y}^{(i)}\left(\mathbf{y}^{(i)}\right)^{H}\right]$ are determined as

$$
E\left[\mathbf{h}^{(i)}\left(\mathbf{y}^{(i)}\right)^{H}\right]=\mathbf{R}_{\mathbf{h}}^{(i)} \mathbf{X}^{H}
$$

and

$$
E\left[\mathbf{y}^{(i)}\left(\mathbf{y}^{(i)}\right)^{H}\right]=\mathbf{X} \mathbf{R}_{\mathbf{h}}^{(i)} \mathbf{X}^{H}+\sigma_{w}^{2} \mathbf{I}_{N}
$$

where $\mathbf{R}_{\mathbf{h}}^{(i)}=E\left[\mathbf{h}^{(i)}\left(\mathbf{h}^{(i)}\right)^{H}\right]$. Using (4), $\mathbf{R}_{\mathbf{h}}^{(i)}$ is written as

$$
\mathbf{R}_{\mathbf{h}}^{(i)}=\mathbf{S}_{o} E\left[\mathbf{f}^{(i)}\left(\mathbf{f}^{(i)}\right)^{H}\right] \mathbf{S}_{o}^{H}=\mathbf{S}_{o} \mathbf{R}_{\mathbf{f}}^{(i)} \mathbf{S}_{o}^{H} .
$$

Substituting (9) and (10) in (8), the estimation of CFR vector $\mathbf{h}^{(i)}$ is given by

$$
\hat{\mathbf{h}}^{(i)}=\mathbf{R}_{\mathbf{h}}^{(i)} \mathbf{X}^{H}\left(\mathbf{X R}_{\mathbf{h}}^{(i)} \mathbf{X}^{H}+\sigma_{w}^{2} \mathbf{I}_{N}\right)^{-1} \mathbf{y}^{(i)} .
$$

The MSE in estimating the CFR vector $\mathbf{h}^{(i)}$ is defined as

$$
h_{\mathrm{MSE}}^{(i)}=\operatorname{Tr}\left(E\left[\left\|\mathbf{h}^{(i)}-\hat{\mathbf{h}}^{(i)}\right\|^{2}\right]\right) .
$$

Substituting (11) in (12), MSE is derived as

$$
h_{\mathrm{MSE}}^{(i)}=\operatorname{Tr}\left(\mathbf{R}_{\mathbf{h}}^{(i)}-\mathbf{R}_{\mathbf{h}}^{(i)} \mathbf{X}^{H}\left(\mathbf{X} \mathbf{R}_{\mathbf{h}}^{(i)} \mathbf{X}^{H}+\sigma_{w}^{2} \mathbf{I}_{N}\right)^{-1} \mathbf{X R}_{\mathbf{h}^{(i)}}\right) .
$$

Using matrix inversion lemma, $\mathbf{A}^{-1}-\mathbf{A}^{-1} \mathbf{B}\left(\mathbf{D} \mathbf{A}^{-1} \mathbf{B}+\mathbf{C}^{-1}\right)^{-1} \mathbf{D} \mathbf{A}^{-1}=(\mathbf{A}+\mathbf{B C D})^{-1}, \quad$ and assuming $\mathbf{A}=\left(\mathbf{R}_{\mathbf{h}}^{(i)}\right)^{-1}, \mathbf{B}=\mathbf{X}^{H}, \mathbf{C}=\sigma_{w}^{-2} \mathbf{I}_{N}$ and $\mathbf{D}=\mathbf{X}$, (13) is simplified as

$$
h_{\mathrm{MSE}}^{(i)}=\operatorname{Tr}\left(\left(\sigma_{w}^{-2} \mathbf{X}^{H} \mathbf{X}+\left(\mathbf{R}_{\mathbf{h}}^{(i)}\right)^{-1}\right)^{-1}\right)
$$

On rearranging, (14) is rewritten as

$$
h_{\mathrm{MSE}}^{(i)}=\operatorname{Tr}\left(\mathbf{R}_{\mathbf{h}}^{(i)}\left(\sigma_{w}^{-2} \mathbf{R}_{\mathbf{h}}^{(i)} \mathbf{X}^{H} \mathbf{X}+\mathbf{I}_{N}\right)^{-1}\right) .
$$

As $\mathbf{R}_{\mathbf{h}}{ }^{(i)}$ is a Hermitian and positive definite matrix and $\mathbf{X}^{H} \mathbf{X}$ in (15) is a multiple of the identity matrix, $\left(\sigma_{w}^{-2} \mathbf{R}_{\mathbf{h}}^{(i)} \mathbf{X}^{H} \mathbf{X}+\mathbf{I}_{N}\right)$ is also Hermitian and positive definite matrix. It has the same basis of eigen decomposition as that of $\mathbf{R}_{\mathbf{h}}{ }^{(i)}$ [21]. The eigen decomposition of $\left(\sigma_{w}^{-2} \mathbf{R}_{\mathbf{h}}^{(i)} \mathbf{X}^{H} \mathbf{X}+\mathbf{I}_{N}\right)$ is written as

$$
\left(\sigma_{w}^{-2} \mathbf{R}_{\mathbf{h}}^{(i)} \mathbf{X}^{H} \mathbf{X}+\mathbf{I}_{N}\right)=\mathbf{U}^{(i)} \bar{\Lambda}^{(i)}\left(\mathbf{U}^{(i)}\right)^{H}
$$

where $\overline{\boldsymbol{\Lambda}}$ is $N \times N$ diagonal matrix with the $r_{c}$ number of non zero diagonal elements $\left[\bar{\lambda}_{0}^{(i)}, \bar{\lambda}_{1}^{(i)}, \ldots, \bar{\lambda}_{r_{c-1}}^{(i)}\right]$. The value of $\bar{\lambda}_{k}^{(i)}$ can be written in terms of $\lambda_{k}^{(i)}$ as

$$
\bar{\lambda}_{k}^{(i)}=\sigma_{w}^{-2} x_{k}^{*} x_{k} \lambda_{k}^{(i)}+1, k=0,1, \ldots, r_{c}-1 .
$$

Let $p_{k}=x_{k}^{*} x_{k}$. The vector expression for MSE in (15) is reduced to scalar expression as

$$
h_{\mathrm{MSE}}^{(i)}=\sum_{k=0}^{r_{c}-1} \frac{\lambda_{k}^{(i)}}{\sigma_{w}^{-2} p_{k} \lambda_{k}^{(i)}+1} .
$$

Mean square error in (18) can be minimized by allocating optimal power to $r_{c}$ subcarriers, with the sum power constraint $\sum_{k=0}^{r_{c}-1} p_{k}=P$ where $P$ is the total power. Mathematically, the optimization problem is formulated as

$\underset{p_{k}}{\operatorname{Minimize}} \mathrm{MSE}=\sum_{k=0}^{r_{c}-1} \frac{\lambda_{k}^{(i)}}{\sigma_{w}^{-2} p_{k} \lambda_{k}^{(i)}+1}$, s.t $\sum_{k=0}^{r_{c}-1} p_{k}=P$.

The equation in (19) is a constrained optimization problem and can be solved using Lagrangian multiplier method. The Lagrangian associated with the minimization problem in (19) is given by

$$
L\left(\mu, p_{k}\right)=\sum_{k=0}^{r_{c}-1} \frac{\lambda_{k}^{(i)}}{\sigma_{w}^{-2} p_{k} \lambda_{k}^{(i)}+1}+\mu\left(\sum_{k=0}^{r_{c}-1} p_{k}-P\right), k=0,1, \ldots, r_{c}-1
$$

where $\mu$ is Lagrangian multiplier.

The optimal power for the $k^{\text {th }}$ subcarrier $p_{k}$ is computed by differentiating (20) with respect to $p_{k}$ and equating to zero. It is determined as

$$
p_{k}^{o p t}=\left(\sqrt{\frac{\sigma_{w}^{2}}{\mu}}-\frac{\sigma_{w}^{2}}{\lambda_{k}^{(i)}}\right)^{+}, \quad k=0,1, \ldots, r_{c}-1
$$

where $(y)^{+}=\max (y, 0)$. Using (21) and the sum power constraint in (19), the expression for Lagrangian multiplier $\mu$ is derived as

$$
\mu=\sigma_{w}^{2} r_{c}^{2}\left(P+\sigma_{w}^{2} \sum_{k=0}^{r_{c}-1} \frac{1}{\lambda_{k}^{(i)}}\right)^{-2} .
$$

Substituting the value of $\mu$ from (22) in (21), the expression for optimal power for the $k^{\text {th }}$ subcarrier that minimizes the mean square error of the LMMSE channel estimator is expressed as

$$
p_{k}^{o p t}=\left(\frac{P}{r_{c}}+\frac{\sigma_{w}^{2}}{r_{c}} \sum_{k=0}^{r_{c}-1} \frac{1}{\lambda_{k}^{(i)}}-\frac{\sigma_{w}^{2}}{\lambda_{k}^{(i)}}\right)^{+}, k=0,1, \ldots, r_{c}-1 .
$$

\section{Performance Analysis with ATSA Error}

This paper deals with MIMO-OFDM system in Time Division Duplexing (TDD) environment. At the transmitter, antenna is selected for each subcarrier based on chan- 
nel coefficients between each transmit antenna and receive antenna. The antenna selection information is transmitted to the receiver through a binary symmetric control channel. However, when the bandwidth of binary symmetric control channel is limited, it introduces ATSA error. Conventionally, LMMSE estimator is employed at the receiver to estimate the channel coefficients at the receiver. Although it performs well at low SNR, it introduces irreducible error at high SNR in the presence of ATSA error. This section characterizes the MSE due to ATSA error. Let $\mathbf{S}_{o, k}$ be $N \times N n_{t}$ selection matrix with error in the $k^{\text {th }}$ subcarrier. The resultant CFR vector is $\mathbf{h}_{k}^{(i)}=\mathbf{S}_{o, k} \mathbf{f}^{(i)}$. The correlation matrix of $\mathbf{h}_{k}^{(i)}$ is $\mathbf{R}_{\mathbf{h}, k}^{(i)}=\mathbf{S}_{o, k} E\left[\mathbf{f}^{(i)}\left(\mathbf{f}^{(i)}\right)^{H}\right] \mathbf{S}_{o, k}^{H}=\mathbf{S}_{o, k} \mathbf{R}_{\mathbf{f}}^{(i)} \mathbf{S}_{o, k}^{H}$. Then, LMMSE channel estimate in (11) is modified as

$$
\hat{\mathbf{h}}_{k}^{(i)}=\mathbf{R}_{\mathbf{h}, k}^{(i)} \mathbf{X}^{H}\left(\mathbf{X R}_{\mathbf{h}, k}^{(i)} \mathbf{X}^{H}+\sigma_{w}^{2} \mathbf{I}_{N}\right)^{-1} \mathbf{y}^{(i)} .
$$

Using simple algebraic metric, the LMMSE estimate in (24) is modified as [20]

$$
\hat{\mathbf{h}}_{k}^{(i)}=\mathbf{R}_{\mathbf{h}, k}^{(i)} \mathbf{X}^{H} \mathbf{X}^{-1}\left(\mathbf{R}_{\mathbf{h}, k}^{(i)}+\sigma_{w}^{2}\left(\mathbf{X} \mathbf{X}^{H}\right)^{-1}\right)^{-1} \mathbf{X}^{-H} \mathbf{y}^{(i)} .
$$

With real data as pilots, (25) is rewritten as,

$$
\hat{\mathbf{h}}_{k}^{(i)}=\mathbf{R}_{\mathbf{h}, k}^{(i)}\left(\mathbf{R}_{\mathbf{h}, k}^{(i)}+\sigma_{w}^{2}\left(\mathbf{X X} \mathbf{X}^{H}\right)^{-1}\right)^{-1} \mathbf{X}^{-1} \mathbf{y}^{(i)}
$$

The mean square error of LMMSE estimator with ATSA error in the $k^{\text {th }}$ subcarrier is written as

$$
\begin{aligned}
h_{\mathrm{MSE}(k, A T S A)}^{(i)} & =E\left[\left\|\mathbf{h}^{(i)}-\hat{\mathbf{h}}_{k}^{(i)}\right\|^{2}\right] \\
& =E\left[\left(\mathbf{h}^{(i)}-\hat{\mathbf{h}}_{k}^{(i)}\right)\left(\mathbf{h}^{(i)}\right)^{H}-\left(\mathbf{h}^{(i)}-\hat{\mathbf{h}}_{k}^{(i)}\right)\left(\hat{\mathbf{h}}_{k}^{(i)}\right)^{H}\right] .
\end{aligned}
$$

According to orthogonality principle, the second term in (27) is zero [19]. Then, $h_{\mathrm{MSE}(k, A T S A)}^{(i)}$ is given by

$$
h_{\mathrm{MSE}(k, A T S A)}^{(i)}=E\left[\left(\mathbf{h}^{(i)}-\hat{\mathbf{h}}_{k}^{(i)}\right)\left(\mathbf{h}^{(i)}\right)^{H}\right] \text {. }
$$

Substituting (26) in (28), $h_{\mathrm{MSE}(k, A T S A)}^{(i)}$ is determined as

$$
\begin{aligned}
& h_{\mathrm{MSE}(k, A T S A)}^{(i)}= \\
& \quad=\operatorname{Tr}\left(\mathbf{R}_{\mathbf{h}}^{(i)}-\sigma_{w}^{-2} \mathbf{R}_{\mathbf{h}}^{(i)} \mathbf{R}_{\mathbf{h}, k}^{(i)} \mathbf{X} \mathbf{X}^{H}\left(\sigma_{w}^{-2} \mathbf{R}_{\mathbf{h}, k}^{(i)}\left(\mathbf{X} \mathbf{X}^{H}\right)+\mathbf{I}_{N}\right)^{-1}\right)
\end{aligned}
$$

The expression for $h_{\mathrm{MSE}(k, A T S A)}^{(i)}$ in (29) can be simplified further using eigenvalue decomposition of the terms $\mathbf{R}_{\mathbf{h}, k}^{(i)} \quad$ and $\sigma_{w}^{-2} \mathbf{R}_{\mathbf{h}, k}^{(i)}\left(\mathbf{X X}^{H}\right)+\mathbf{I}_{N}$. The EVD of $\mathbf{R}_{\mathbf{h}, k}^{(i)}$ is $\mathbf{U}_{k}^{(i)} \boldsymbol{\Lambda}_{k}^{(i)}\left(\mathbf{U}_{k}^{(i)}\right)^{H}$ where $\mathbf{U}_{k}^{(i)}$ is a unitary matrix and $\boldsymbol{\Lambda}_{k}^{(i)}$ is
$N \times N$ diagonal matrix with its eigenvalues as diagonal elements. $\mathbf{X} \mathbf{X}^{H}$ in (29) is a multiple of the identity matrix. Then, $\left(\sigma_{w}^{-2} \mathbf{R}_{\mathbf{h}, k}^{(i)} \mathbf{X} \mathbf{X}^{H}+\mathbf{I}_{N}\right)$ is also Hermitian and positive definite matrix and it has the same basis of eigen decomposition as that of $\mathbf{R}_{\mathbf{h}, k}^{(i)}$ [21]. Hence, the eigen decomposition of $\left(\sigma_{w}^{-2} \mathbf{R}_{\mathbf{h}, k}^{(i)} \mathbf{X} \mathbf{X}^{H}+\mathbf{I}_{N}\right)$ can be written as

$$
\left(\sigma_{w}^{-2} \mathbf{R}_{\mathbf{h}, k}^{(i)} \mathbf{X} \mathbf{X}^{H}+\mathbf{I}_{N}\right)=\mathbf{U}_{k}^{(i)} \bar{\Lambda}_{k}^{(i)}\left(\mathbf{U}_{k}^{(i)}\right)^{H}
$$

where $\bar{\Lambda}_{k}^{(i)}$ is the diagonal matrix with its $j^{\text {th }}$ diagonal element as

$$
\bar{\lambda}_{k, j}^{(i)}=\sigma_{w}^{-2} x_{j}^{*} x_{j} \lambda_{k, j}^{(i)}+1, \quad j=0,1, \ldots, r_{k}-1
$$

where $r_{k}$ is the rank of $\mathbf{R}_{\mathbf{h}, k}^{(i)}$. Substituting (30) in (29), $h_{\mathrm{MSE}(k, A T S A)}^{(i)}$ is written as

$$
\begin{aligned}
h_{\operatorname{MSE}(k, A T S A)}^{(i)}= & \sum_{j=0}^{r_{c}-1} \lambda_{j}^{(i)}- \\
& \sigma_{w}^{-2} \operatorname{Tr}\left(\left(\mathbf{U}_{k}^{(i)}\right)^{H} \mathbf{R}_{\mathbf{h}}^{(i)} \mathbf{R}_{\mathbf{h}, k}^{(i)} \mathbf{X} \mathbf{X}^{H} \mathbf{U}_{k}^{(i)}\left(\bar{\Lambda}_{k}^{(i)}\right)^{-1}\right)
\end{aligned}
$$

where $r_{c}$ is the rank of $\mathbf{R}_{\mathbf{h}}{ }^{(i)}$. Let

$$
\left(\mathbf{U}_{k}^{(i)}\right)^{H} \mathbf{R}_{\mathbf{h}}^{(i)} \mathbf{R}_{\mathbf{h}, k}^{(i)} \mathbf{X} \mathbf{X}^{H} \mathbf{U}_{k}^{(i)}=\mathbf{W}_{k}^{(i)}
$$

Using (31) and (33), (32) is simplified as

$$
h_{\mathrm{MSE}(k, A T S A)}^{(i)}=\sum_{j=0}^{r_{c}-1} \lambda_{j}^{(i)}-\sum_{j=0}^{r_{k}-1}\left(\frac{\left(\mathbf{W}_{k}^{(i)}\right)_{j j}}{\lambda_{k . j}^{(i)} x_{j} x_{j}^{*}+\sigma_{w}^{2}}\right) .
$$

With perfect ATSA, replacing $\mathbf{R}_{\mathbf{h}, k}^{(i)}$ with $\mathbf{R}_{\mathbf{h}}^{(i)}$ in (29) and simplifying gives an expression for mean square error with perfect ATSA in the same format as (34). It is derived as,

$$
h_{\mathrm{MSE}(c)}^{(i)}=\sum_{j=0}^{r_{c}-1} \lambda_{j}^{(i)}-\sum_{j=0}^{r_{c}-1}\left(\frac{\left(\mathbf{W}_{c}^{(i)}\right)_{j j}}{\lambda_{j}^{(i)} x_{j} x_{j}^{*}+\sigma_{w}^{2}}\right)
$$

where $\mathbf{W}_{c}^{(i)}=\left(\Lambda^{(i)}\right)^{2}\left(\mathbf{U}^{(i)}\right)^{H} \mathbf{X} \mathbf{X}^{H} \mathbf{U}^{(i)}$. When a binary symmetric channel with the crossover probability of $q$ is utilized to send the selection information in $\mathbf{S}_{o}$, the probability of receiving a symbol with no ATSA error is $\frac{q_{1}}{q_{1}+q_{2} r_{c}}$ [13], where $q_{1}=(1-q)^{r_{c}}$ and $q_{2}=q(1-q)^{r_{c}-1}$. The probability of receiving a symbol with error at the $k^{\text {th }}$ subcarrier is $\frac{q_{2}}{q_{1}+q_{2} r_{c}}$. Then, the average mean square 
error of LMMSE estimator with crossover probability $q$ of binary symmetric channel is given by

$$
h_{\mathrm{MSE}(q)}^{(i)}=\frac{q_{1}}{q_{1}+q_{2} r_{c}} h_{\mathrm{MSE}(c)}^{(i)}+\frac{q_{2}}{q_{1}+q_{2} r_{c}} \sum_{k=1}^{r_{c}} h_{\mathrm{MSE}(k, A T S A)}^{(i)} .
$$

Substituting (34) and (35) in (36) and replacing $x_{j} x_{j}^{*}$ by the optimal power $p_{j}$ derived in Sec. $3, h_{\operatorname{MSE}(q)}^{(i)}$ is given by

$$
\begin{aligned}
& h_{\mathrm{MSE}(q)}^{(i)}=\bar{q}_{1}\left(\sum_{j=0}^{r_{c}-1} \lambda_{j}^{(i)}-\sum_{j=0}^{r_{c}-1}\left(\frac{\left(\mathbf{W}_{c}^{(i)}\right)_{j j}}{\lambda_{j}^{(i)} p_{j}+\sigma_{w}^{2}}\right)\right)+ \\
& \bar{q}_{2} \sum_{k=1}^{r_{c}}\left(\sum_{j=0}^{r_{c}-1} \lambda_{j}^{(i)}-\sum_{j=0}^{r_{k}-1}\left(\frac{\left(\mathbf{W}_{k}^{(i)}\right)_{j j}}{\lambda_{k, j}^{(i)} p_{j}+\sigma_{w}^{2}}\right)\right)
\end{aligned}
$$

where $\bar{q}_{1}=\frac{q_{1}}{q_{1}+q_{2} r_{c}}$ and $\bar{q}_{2}=\frac{q_{2}}{q_{1}+q_{2} r_{c}}$.

Multiplying each element within summation in second term and fourth term of (37) by $\left(\lambda_{j}^{(i)} p_{j}-\sigma_{w}^{2}\right)$ and $\left(\lambda_{k, j}^{(i)} p_{j}-\sigma_{w}^{2}\right)$ respectively and ignoring the term $\sigma_{w}^{4}$ to get high SNR approximation results in

$$
\begin{aligned}
& h_{\operatorname{MSE}(q)}^{(i)}=\bar{q}_{1} \sum_{j=0}^{r_{c}-1} \lambda_{j}^{(i)}-\bar{q}_{1} \sum_{j=0}^{r_{c}-1} \frac{\left(\mathbf{W}_{c}^{(i)}\right)_{j j}}{\lambda_{j}^{(i)} p_{j}}+\bar{q}_{1} \sigma_{w}^{2} \sum_{j=0}^{r_{c}-1} \frac{\left(\mathbf{W}_{c}^{(i)}\right)_{j j}}{\left(\lambda_{j}^{(i)} p_{j}\right)^{2}}+ \\
& \bar{q}_{2} \sum_{k=1}^{r_{c}} \sum_{j=0}^{r_{c}-1} \lambda_{j}^{(i)}-\bar{q}_{2} \sum_{k=1}^{r_{c}} \sum_{j=0}^{r_{k}-1} \frac{\left(\mathbf{W}_{k}^{(i)}\right)_{j j}}{\lambda_{k, j}^{(i)} p_{j}}+\bar{q}_{2} \sigma_{w}^{2} \sum_{k=1}^{r_{c}} \sum_{j=0}^{r_{k}-1} \frac{\left(\mathbf{W}_{k}^{(i)}\right)_{j j}}{\left(\lambda_{k, j}^{(i)} p_{j}\right)^{2}} .
\end{aligned}
$$

\section{Proposed Hybrid Estimator with Optimal Power Allocation}

The performance degradation of LMMSE estimator due to the error in selection information can be overcome only if the correlation matrix $\mathbf{R}_{\mathbf{h}}{ }^{(i)}$ is independent of selection matrix. If suppose, the LMMSE estimator is relaxed to use the identity matrix instead of correlation matrix $\mathbf{R}_{\mathbf{h}}{ }^{(i)}$, then the performance degradation can be minimized significantly [22]. In (11), substituting $\mathbf{R}_{\mathbf{h}}{ }^{(i)}=\alpha \mathbf{I}$, the relaxed MMSE estimate is derived as

$$
\hat{\mathbf{h}}_{R}^{(i)}=\mathbf{X}^{H}\left(\mathbf{X} \mathbf{X}^{H}+\sigma_{w}^{2} \mathbf{I}_{N}\right)^{-1} \mathbf{y}^{(i)}
$$

By minimizing $E\left(\left\|\mathbf{h}^{(i)}-\hat{\mathbf{h}}_{R}^{(i)}\right\|^{2}\right)$, mean square error of the relaxed estimator is derived as

$$
h_{\mathrm{MSE}(R)}^{(i)}=\sum_{j=0}^{r_{c}-1} \lambda_{j}^{(i)}-\sum_{j=0}^{r_{c}-1} \frac{x_{j} x_{j}^{*} \lambda_{j}^{(i)}}{x_{j} x_{j}^{*}+\sigma_{w}^{2}} .
$$

Replacing $x_{j} x_{j}^{*}$ by optimal power $p_{j}$ derived in Sec. 3, (40) is rewritten as

$$
h_{\mathrm{MSE}(R)}^{(i)}=\sum_{j=0}^{r_{c}-1} \lambda_{j}^{(i)}-\sum_{j=0}^{r_{c}-1} \frac{p_{j} \lambda_{j}^{(i)}}{p_{j}+\sigma_{w}^{2}} .
$$

Multiplying and dividing the term $\left(p_{j}-\sigma_{w}^{2}\right)$ inside the summation of the second term, and ignoring the term $\left(\sigma_{w}^{2}\right)^{2},(41)$ becomes

$$
h_{\mathrm{MSE}(R)}^{(i)}=\sigma_{w}^{2} \sum_{j=0}^{r_{c}-1} \frac{\lambda_{j}^{(i)}}{p_{j}} .
$$

It is observed that $h_{\mathrm{MSE}(R)}^{(i)}$ is larger compared to $h_{\mathrm{MSE}(q)}^{(i)}$ of LMMSE estimator at low SNR, as it depends only on diagonal elements of the correlation matrix. Its value decreases with increasing SNR, where LMMSE estimator gives irreducible error. Combining the merits of both LMMSE and RMMSE estimators, a hybrid estimator is proposed. It performs as LMMSE estimator till the noise dominates the effect of ATSA error. The proposed estimator switches to RMMSE estimator when $h_{\mathrm{MSE}(q)}^{(i)}$ of LMMSE estimator becomes $\sigma_{w}^{2} \sum_{j=0}^{r_{c}-1} \frac{\lambda_{j}^{(i)}}{p_{j}}$. The receive SNR per-subcarrier at which hybrid estimator switches from LMMSE to RMMSE is determined from

$$
h_{\mathrm{MSE}(q)}^{(i)}=h_{\mathrm{MSE}(R)}^{(i)} .
$$

Substituting (38) and (42) in (43), it is rewritten as

$$
\begin{aligned}
& \bar{q}_{1} \sum_{j=0}^{r_{c}-1} \lambda_{j}^{(i)}-\bar{q}_{1} \sum_{j=0}^{r_{c}-1} \frac{\left(\mathbf{W}_{c}^{(i)}\right)_{j j}}{\lambda_{j}^{(i)} p_{j}}+\bar{q}_{1} \sigma_{w}^{2} \sum_{j=0}^{r_{c}-1} \frac{\left(\mathbf{W}_{c}^{(i)}\right)_{j j}}{\left(\lambda_{j}^{(i)} p_{j}\right)^{2}}+\bar{q}_{2} \sum_{k=1}^{r_{c}} \sum_{j=0}^{r_{c}-1} \lambda_{j}^{(i)} \\
& -\bar{q}_{2} \sum_{k=1}^{r_{c}} \sum_{j=0}^{r_{k}-1} \frac{\left(\mathbf{W}_{k}^{(i)}\right)_{j j}}{\lambda_{k, j}^{(i)} p_{j}}+\bar{q}_{2} \sigma_{w}^{2} \sum_{k=1}^{r_{c}} \sum_{j=0}^{r_{k}-1} \frac{\left(\mathbf{W}_{k}^{(i)}\right)_{j j}}{\left(\lambda_{k, j}^{(i)} p_{j}\right)^{2}}=\sigma_{w}^{2} \sum_{j=0}^{r_{c}-1} \frac{\lambda_{j}^{(i)}}{p_{j}} .(44)
\end{aligned}
$$

Replacing $\sigma_{w}^{2}$ by $1 / \beta$ and solving for $\beta$ results in

$$
\mathrm{SNR}_{T H}=\beta=\frac{c_{3}-\bar{q}_{1} c_{4}-\bar{q}_{2} c_{5}}{\bar{q}_{1} c_{1}+\bar{q}_{2} c_{2}}
$$

where

$$
\begin{aligned}
& c_{1}=\sum_{j=0}^{r_{c}-1} \lambda_{j}^{(i)}-\sum_{j=0}^{r_{c}-1} \frac{\left(\mathbf{W}_{c}^{(i)}\right)_{j j}}{\lambda_{j}^{(i)} p_{j}}, \quad c_{2}=r_{c} \sum_{j=0}^{r_{c}-1} \lambda_{j}^{(i)}-\sum_{k=1}^{r_{c}} \sum_{j=0}^{r_{k}-1} \frac{\left(\mathbf{W}_{k}^{(i)}\right)_{j j}}{\lambda_{k, j}^{(i)} p_{j}}, \\
& c_{3}=\sum_{j=0}^{r_{c}-1} \frac{\lambda_{j}^{(i)}}{p_{j}}, \quad c_{4}=\sum_{j=0}^{r_{c}-1} \frac{\left(\mathbf{W}_{c}^{(i)}\right)_{j j}}{\left(\lambda_{j}^{(i)} p_{j}\right)^{2}}, \quad c_{5}=\sum_{k=1}^{r_{c}} \sum_{j=0}^{r_{k}-1} \frac{\left(\mathbf{W}_{k}^{(i)}\right)_{j j}}{\left(\lambda_{k, j}^{(i)} p_{j}\right)^{2}} .
\end{aligned}
$$




\section{Results and Discussion}

In this section, simulations are carried out to analyze the MSE performance of the proposed hybrid channel estimator with optimal power allocation in the presence of ATSA error in MIMO-OFDM system with per-subcarrier antenna selection. The simulation parameters are listed in Tab. 1 .

\begin{tabular}{|c|c|c|}
\hline $\begin{array}{r}\text { Sl. } \\
\text { No }\end{array}$ & Parameters & Values \\
\hline & No. of transmit antennas $\left(n_{t}\right)$ & 2 \\
\hline & No. of receive antennas $\left(n_{r}\right)$ & 2 \\
\hline & Sampling frequency & $30.72 \mathrm{MHz}$ \\
\hline & Channel power delay profile & $\begin{array}{c}\text { Uniform, } \\
\text { Extended Pedestrian-A, } \\
\text { Extended Vehicular-A }\end{array}$ \\
\hline & Length of channel $(L)$ & $\begin{array}{c}4 \text { (Uniform) } \\
14 \text { (Extended Pedestrian-A) [22] } \\
\end{array}$ \\
& No. of subcarriers $(N)$ & $\begin{array}{c}\text { (Extended Vehicular- A) } \\
\text { 64 (Extended Pedestrian-A) } \\
128 \text { (Extended Vehicular- A) }\end{array}$ \\
\hline
\end{tabular}

Tab. 1. Simulation parameters for MIMO-OFDM system with per-subcarrier antenna selection with optimal power allocation.

The effect of ATSA error in average MSE is studied by plotting the normalized error, which is defined as $\eta=\left|h_{\mathrm{MSE}(c)}^{(i)}-h_{\mathrm{MSE}(q)}^{(i)}\right| / h_{\mathrm{MSE}(c)}^{(i)}$. Figure 1 shows the effect of ATSA error in LMMSE estimator for the control channel crossover probabilities of 0.1 and 0.01 , in a $2 \times 2$ MIMOOFDM system with per-subcarrier transmit antenna selection. The number of subcarriers in the OFDM symbol is 16. With the crossover probability of $q=0.1, \eta$ is 0.2592 at SNR of $10 \mathrm{~dB}$ and it increases to 7.417 at SNR of $30 \mathrm{~dB}$. Similarly, when $q=0.01, \eta$ increases from 0.02985 at $10 \mathrm{~dB}$ to 0.8685 at $30 \mathrm{~dB}$. As the normalized error increases with increase in SNR, performance of LMMSE estimator decreases as SNR increases.

Figure 2 shows the MSE performance of LMMSE estimator with perfect ATSA, when the proposed optimal

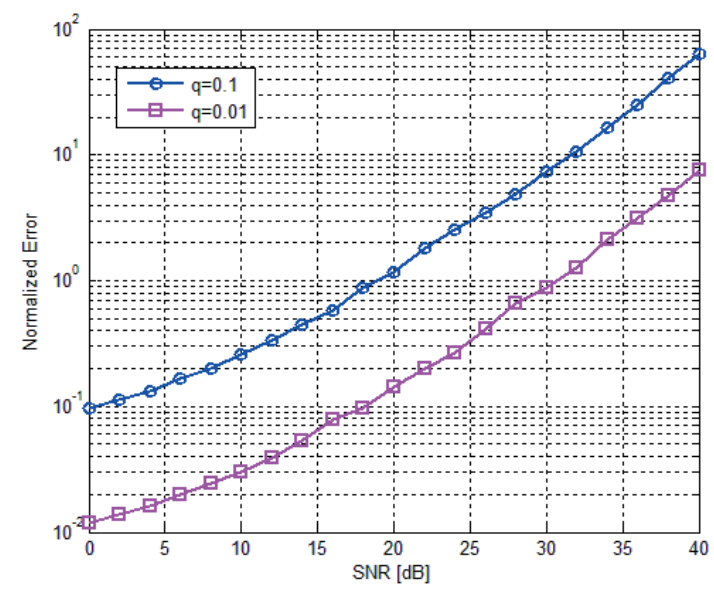

Fig. 1. Effect of ATSA error in the performance of LMMSE estimation with optimal power allocation.

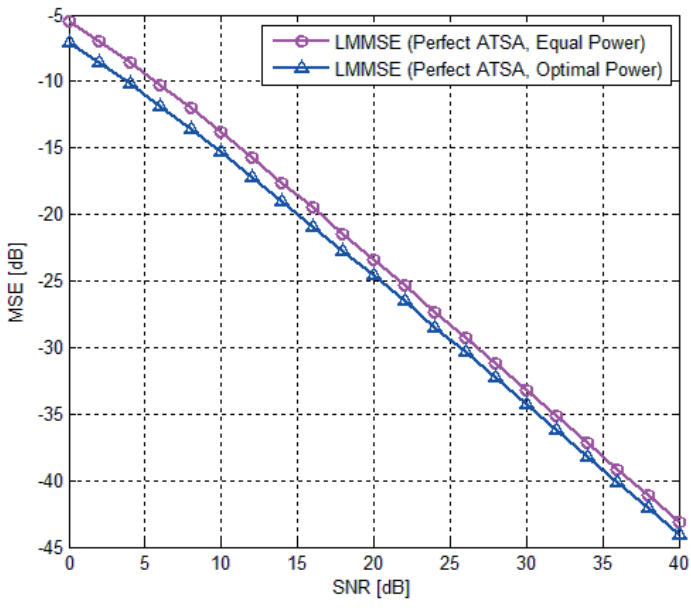

Fig. 2. MSE performance of LMMSE estimator with optimal and equal power allocation.

power allocation is applied at subcarrier level in $2 \times 2$ MIMO-OFDM system with per-subcarrier transmit antenna selection. It is assumed that the number of subcarriers in the OFDM signal is 16 . The length of the channel is $L=4$. The performance of the estimator is compared with MSE performance of the LMMSE estimator with equal power allocation. It is observed that the LMMSE estimator with optimal power allocation requires $1.2 \mathrm{~dB}$ less SNR compared to LMMSE estimator with equal power allocation at the MSE of $-30 \mathrm{~dB}$.

Figure 3 shows the MSE performance of the proposed hybrid estimator with optimal power allocation. The MSE performance of the proposed hybrid estimator is compared with MSE performances of LMMSE estimator with perfect ATSA, LMMSE estimator with ATSA error and RMMSE estimator with optimal power allocation. It is assumed that the crossover probability of binary symmetric channel is, $q=0.1$ which introduces ATSA error. The number of subcarriers used in OFDM signal is $N=16$ and the channel length is taken as $L=4$. The performance of RMMSE estimator is better than that of LMMSE with ATSA error, at high SNR. As the proposed hybrid estimator is designed such that it combines the merits of both LMMSE and

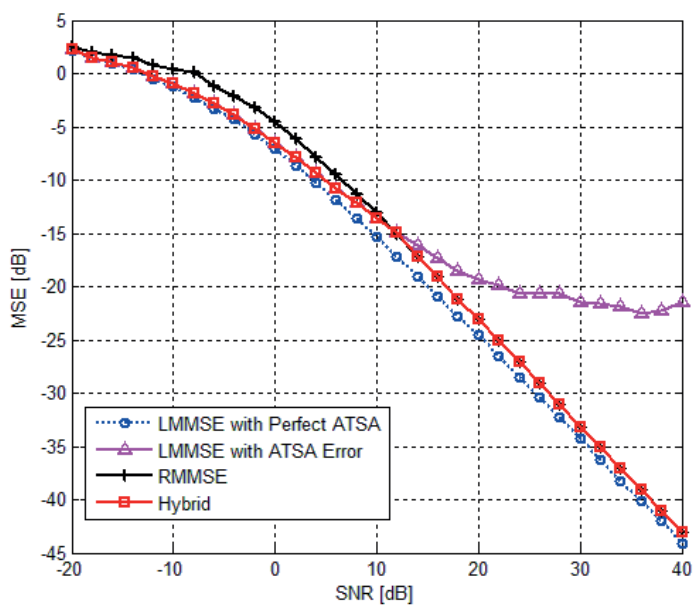

Fig. 3. MSE performance of estimators for $q=0.1$ and $N=16$ with optimal power allocation. 
RMMSE estimators, the MSE performance of the proposed hybrid estimator is same as LMMSE estimator up to $2 \mathrm{~dB}$ SNR. At $2 \mathrm{~dB}$, the performance of LMMSE estimator starts degrading. Further, MSE of LMMSE estimator becomes irreducible and almost constant from the SNR of $12 \mathrm{~dB}$. The proposed hybrid estimator switches to RMMSE estimator at $12 \mathrm{~dB}$. The threshold $\mathrm{SNR}, S N R_{\mathrm{TH}}$ is calculated using (45) for various crossover probabilities of binary symmetric channel assuming that the number of subcarriers is $N=16$ in a $2 \times 2$ MIMO-OFDM system with per-subcarrier antenna selection and with optimal power allocation. They are summarized in Tab. 2. At the threshold SNR, the proposed hybrid estimator switches from LMMSE estimator to RMMSE estimator to improve the MSE performance.

\begin{tabular}{|c|c|c|c|c|c|c|c|}
\hline $\begin{array}{l}\text { Crossover } \\
\text { probability, } \\
q \\
\end{array}$ & 0.1 & 0.09 & 0.07 & 0.05 & 0.03 & 0.01 & 0.001 \\
\hline \begin{tabular}{|c} 
With optimal \\
power \\
allocation \\
SNR \\
threshold \\
$S N R_{\mathrm{TH}}(\mathrm{dB})$ \\
\end{tabular} & 12 & 12.5 & 13 & 14 & 16 & 18 & 26 \\
\hline $\begin{array}{c}\text { With equal } \\
\text { power } \\
\text { allocation } \\
\text { SNR } \\
\text { threshold } \\
S N R_{\mathrm{TH}}(\mathrm{dB}) \\
\end{array}$ & 16 & 16.3 & 16.7 & 17 & 18 & 23 & 31 \\
\hline
\end{tabular}

Tab. 2. Threshold SNR for different crossover probabilities $q$ with optimal and equal power allocation.

Figure 4 shows the MSE performance of the proposed hybrid estimator for $q=0.01$ for $N=16$ and 128 with optimal power allocation among subcarriers. The hybrid estimator switches to RMMSE estimator at SNR of $18 \mathrm{~dB}$ and $22 \mathrm{~dB}$ with 16 and 128 subcarriers per OFDM symbol respectively.

Figure 5 shows the MSE performance of LMMSE, LMMSE with ATSA error, RMMSE and hybrid estimators for extended pedestrian-A power delay profile. The crossover probability of binary symmetric channel is assumed as

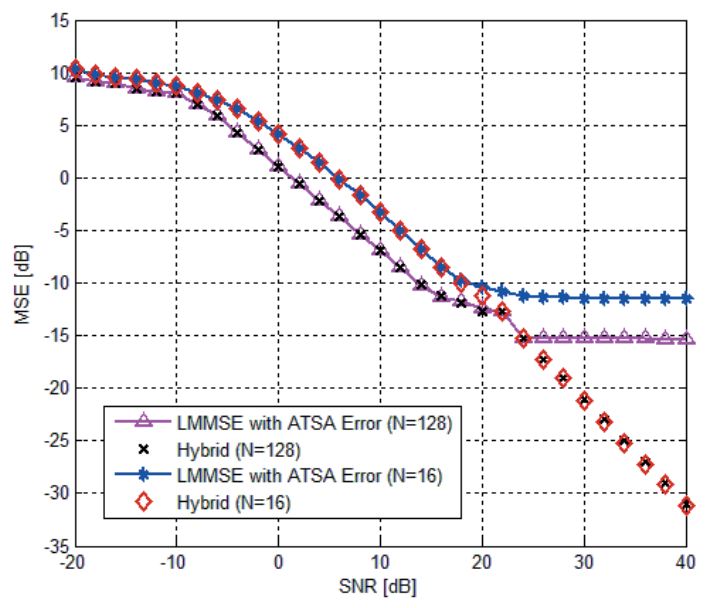

Fig. 4. MSE performance of estimators for $q=0.01$ with optimal ower allocation for different number of subcarriers $N$. $q=0.01$. With use of $30.72 \mathrm{MHz}$ sampling frequency, the length of extended pedestrian-A channel is 14 . Number of subcarriers is to be higher than the length of the channel. Hence 64 subcarriers are used per OFDM symbol. The hybrid estimator switches to RMMSE estimator at SNR of $30 \mathrm{~dB}$.

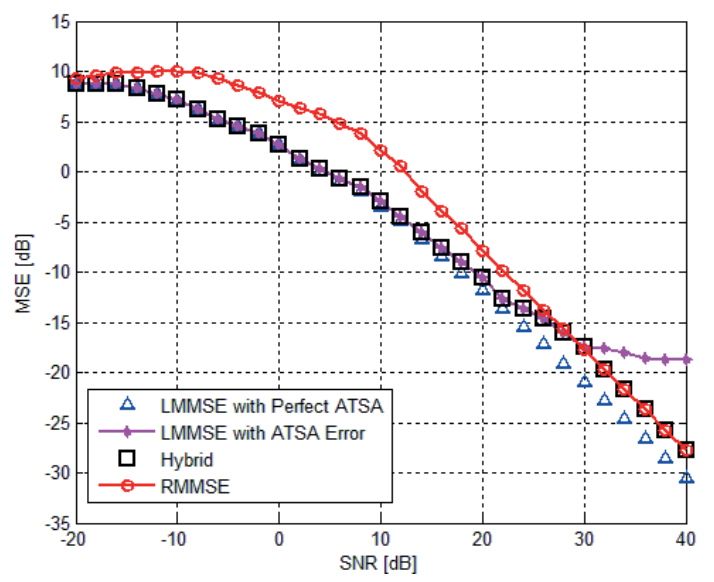

Fig. 5. MSE performance for extended pedestrian-A for $q=0.01$ and $N=64$ with optimal power allocation.

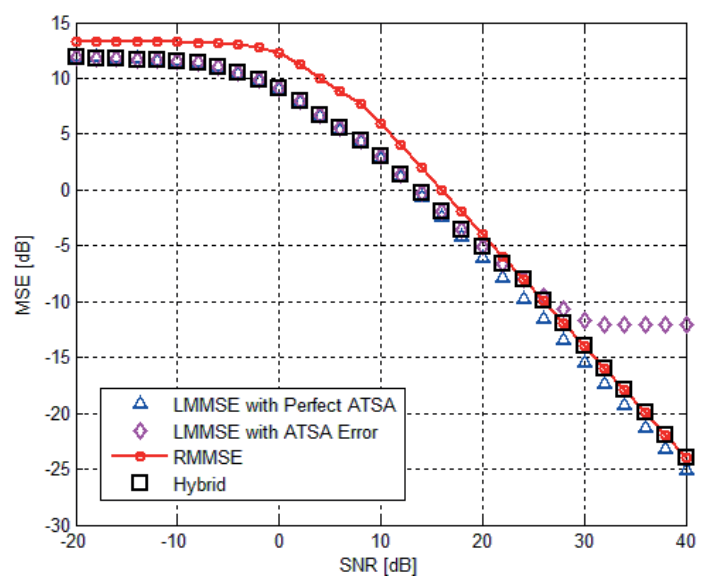

Fig. 6. MSE performance for extended vehicular-A for $q=0.01$ and $N=128$ with optimal power allocation.

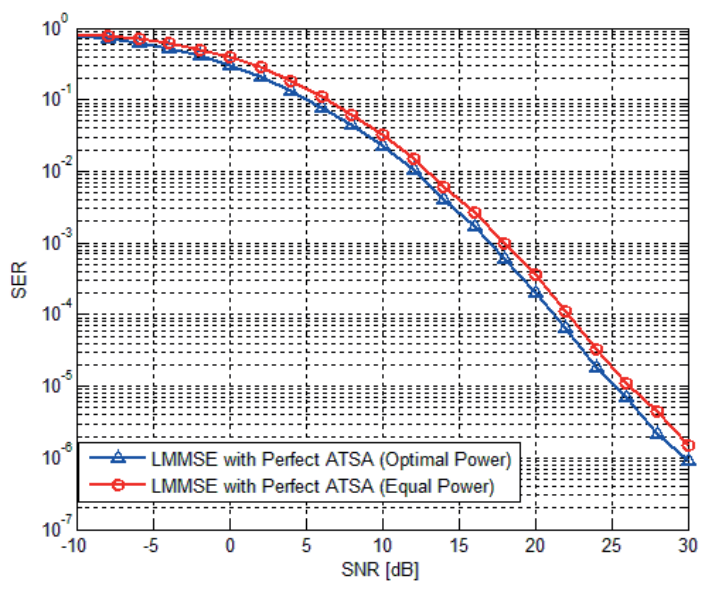

Fig. 7. SER performance of LMMSE estimator for $q=0.1$ and $N=16$ with optimal and equal power allocation. 
Figure 6 shows the MSE performance of LMMSE, LMMSE with ATSA error, RMMSE and hybrid estimators for extended vehicular-A power delay profile. To simulate the MSE performance, an OFDM symbol with 128 subcarriers is considered. The crossover probability of binary symmetric channel is assumed as $q=0.01$. The hybrid estimator switches to RMMSE estimator at SNR of $24 \mathrm{~dB}$.

Figure 7 shows the symbol error rate (SER) performance of LMMSE estimator with perfect ATSA with optimal and equal power allocation in $2 \times 2$ MIMO-OFDM system with per-subcarrier antenna selection. The number of subcarriers in the OFDM signal is considered as 16. The length of the channel is $L=4$. It is assumed that 16-QAM modulation is used. It is observed that the LMMSE estimator with optimal power allocation requires $1 \mathrm{~dB}$ less SNR compared to LMMSE estimator with equal power allocation at the SER of $10^{-3}$.

Figure 8 shows the SER performance of LMMSE estimator with perfect ATSA, LMMSE estimator with ATSA error, RMMSE estimator and proposed hybrid estimator when the crossover probability $q=0.1, N=16$ and $L=4$ with optimal power allocation. LMMSE estimator gives $1 \mathrm{~dB}$ SNR improvement over RMMSE estimator at SER of $10^{-1}$. The performance of RMMSE estimator is better than that of LMMSE with ATSA error, at high SNR. As the proposed hybrid estimator combines the merits of both LMMSE and RMMSE estimators, the SER performance is same as LMMSE estimator for low SNR. SER of LMMSE estimator becomes irreducible after $12 \mathrm{~dB}$. The proposed hybrid estimator switches to RMMSE estimator at $12 \mathrm{~dB}$ exactly.

Figure 9 shows the SER performance of the proposed hybrid estimator for crossover probability $q=0.01$ with number of subcarriers $N=16$ and 128. 16-QAM modulation is used. The hybrid estimator switches to RMMSE estimator at SNR of $18 \mathrm{~dB}$ with 16 subcarriers per OFDM symbol. The hybrid estimator switches to RMMSE estimator at SNR of $22 \mathrm{~dB}$ with 128 subcarriers per OFDM symbol.

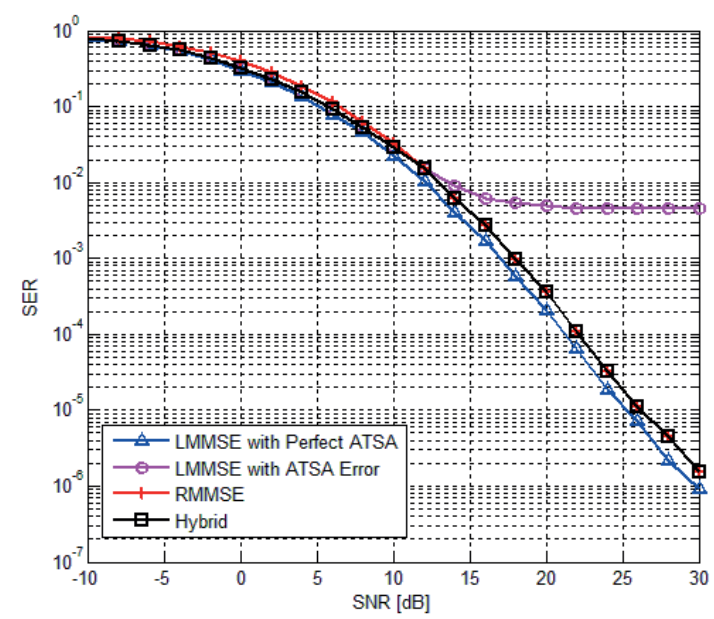

Fig. 8. SER performance of estimators for $q=0.1$ and $N=16$ with optimal power allocation.

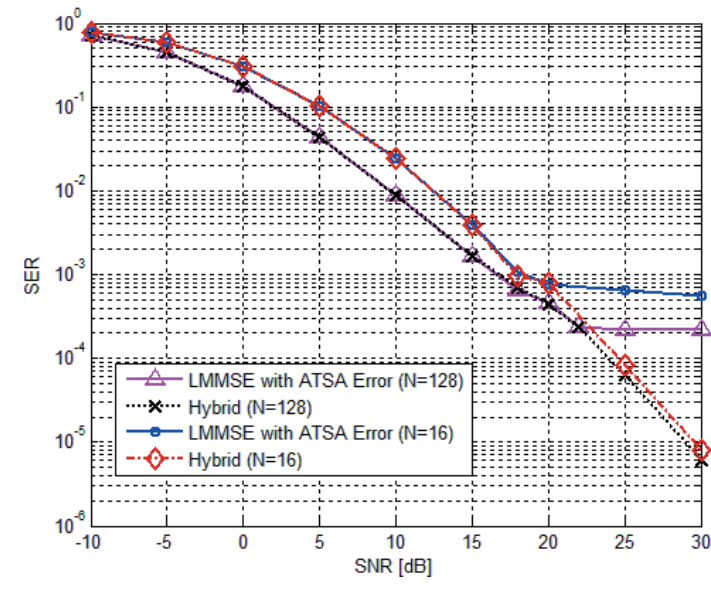

Fig. 9. SER performance of estimators for $q=0.01$.

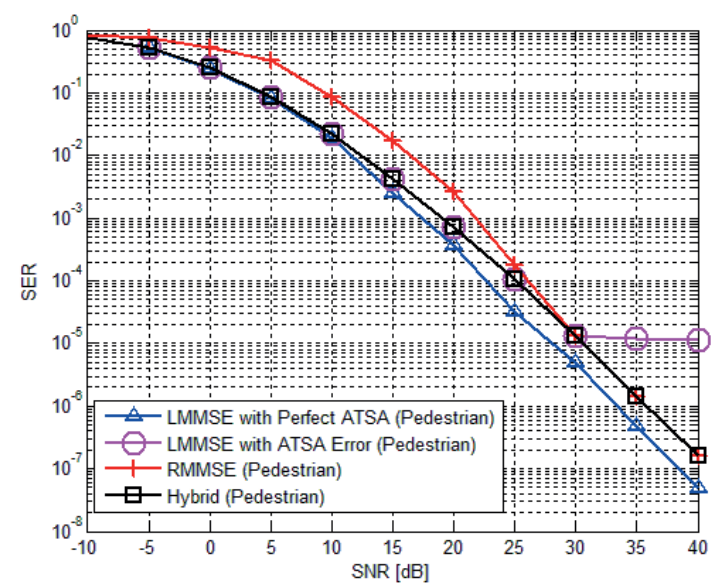

Fig. 10. SER performance for extended pedestrian-A PDP for $q=0.01$ and $N=64$.

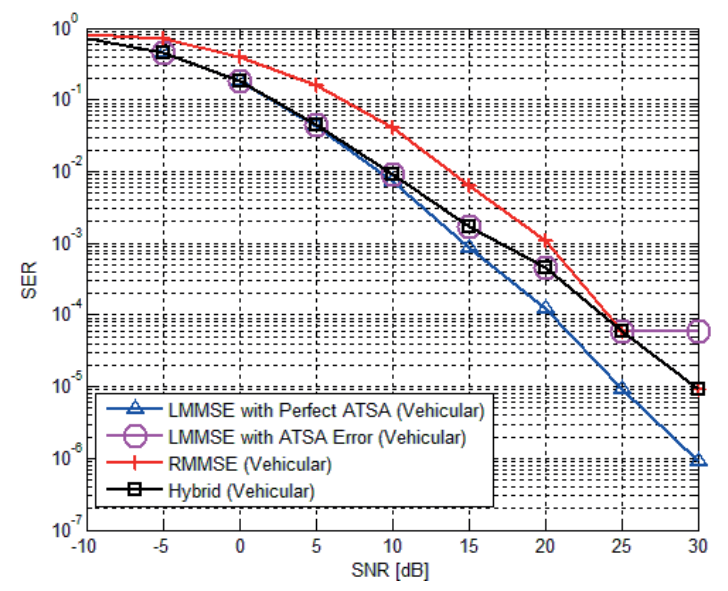

Fig. 11. SER performance for extended vehicular-A PDP for $q=0.01$ and $N=128$.

Figure 10 shows the SER performance of the proposed hybrid estimator with optimal power allocation for extended pedestrian-A power delay profile. It is assumed that the number of subcarriers in the system is $N=64$ and the crossover probability of binary symmetric channel is $q=0.01$. The SER performance with optimal power allocation is compared with SER performances of LMMSE estimator, LMMSE estimator with ATSA error and 
RMMSE estimator. The hybrid channel estimator switches to RMMSE estimator at SNR of $30 \mathrm{~dB}$.

Figure 11 shows the SER performance of LMMSE, LMMSE with ATSA error, RMMSE and hybrid estimators for extended vehicular-A power delay profile. To simulate the SER performance, an OFDM symbol with 128 subcarriers is considered and the crossover probability $q$ is $q=0.01$. The hybrid estimator switches to RMMSE estimator at SNR of $25 \mathrm{~dB}$.

\section{Conclusion}

In this paper, a hybrid channel estimator along with optimal power allocation for per-subcarrier transmit antenna selection in MIMO-OFDM system in the presence of ATSA error is proposed. A scalar MSE expression for the channel estimation is derived to overcome the non invertibility of the autocorrelation matrix of CFR. A convex optimization problem is formulated with an objective to minimize the channel estimation MSE with a sum power constraint. The proposed hybrid channel estimator switches from LMMSE estimator to relaxed MMSE estimator exactly at the SNR where ATSA error dominates. An analytical expression for SNR threshold with optimal power allocation is derived. The SER performance of the proposed hybrid channel estimator is also found to be improved.

\section{References}

[1] STUBER, G. L., BARRY, J. R., MCLAUGHLIN, S. W., YE LI, G., INGRAM, M. A., PRATT, T. G. Broadband MIMO-OFDM wireless communications. In Proceedings of the IEEE, 2004, vol. 92 , no. 2, p. 271-294.

[2] YENG, H. A road to future broadband wireless access: MIMOOFDM based air interface. IEEE Communication Magazine, 2005, vol. 43 , no. 1 , p. 53-60.

[3] ALAMOUTI, S. A simple transmit diversity technique for wireless communications. IEEE Journal on Selected Areas in Communications, 1998, vol. 16, p. 1451-1458.

[4] BOLCSKEI, H., GESBERT, D., PAULRAJ, A. On the capacity of OFDM based spatial multiplexing systems. IEEE Transactions on Communications, 2002, vol. 50, no. 2, p. 225-234. DOI: $10.1109 / 26.983319$

[5] ZHANG, H., NABAR, R. U. Transmit antenna selection in MIMO-OFDM systems: bulk versus per-tone selection. In Proceedings of the IEEE ICC, 2008, p. 4371-4375. DOI: 10.1109/ICC.2008.820

[6] XUN SHAO, JINHONG YUAN, RAPAJIC, P. Antenna selection for MIMO-OFDM spatial multiplexing system. In Proceedings of the International Symposium on Information Theory ISIT 2003. Yokohama (Japan), 2003, p. 90. DOI: 10.1109/ISIT.2003.1228104

[7] Vithanage, C. M., COON, J. P., PARKeR, S. C. J. On capacity-optimal precoding for multiple antenna systems subject to EIRP restrictions. IEEE Transactions on Wireless Communications, 2008 , vol. 7 , no. 12 , p. 5182-5187. DOI: 10.1109/T-WC.2008.070879

[8] COON, J. P., SANDELL, M. Combined bulk and per-tone transmit antenna selection in OFDM systems. IEEE Communications Letters, 2010, vol. 14, no. 5, p. 426-428. DOI: 10.1109/LCOMM.2010.05.100055

[9] COON, J. P., SANDELL, M. Performance of transmit antenna selection in space-time-coded OFDM systems. IEEE Transactions on Vehicular Technology, 2011, vol. 60, no. 6, p. 2824-2828.

[10] VAN DE BEEK, J. J., EDFORS, O., SANDELL, M., WILSON, S. K., BORJESSON, P. O. On channel estimation in OFDM systems. In Proceedings of the $45^{\text {th }}$ IEEE Vehicular Technology Conference. Chicago (IL, USA), 1995, p. 815-819.

[11] LI, Y. G., CIMINI, L. J., SOLLENBERGER, N. R. Robust channel estimation for OFDM systems with rapid dispersive fading channels. IEEE Transactions on Communication, 1998, vol. 46, no. 7, p. 902-915.

[12] SRIVASTAVA, V., CHIN KEONG HO, HO WANG FUNG, P., SUMEI SUN. Robust MMSE channel estimation in OFDM systems with practical timing synchronization. In IEEE Conf. on Wireless Communication and Networking WCNC 2004. Atlanta (USA), 2004, p. 711 - 716. DOI: 10.1109/WCNC.2004.1311273

[13] VITHANAGE, C. M., DENIC, S., SANDELL, M. Robust linear channel estimation methods for per-subcarrier transmit antenna selection. IEEE Transaction on Communications, 2011, vol. 59, no. 7, p. 2018-2028. DOI: 10.1109/TCOMM.2011.051711.100420

[14] RAJESWARI, K., SASHIGANTH, M., THIRUVENGADAM, S.J. A hybrid channel estimator for MIMO-OFDM system with persubcarrier transmit antenna selection. Telecommunication Systems, 2015, vol. 58, no. 1, p. 81-89. DOI: 10.1007/s11235-014-9894-3

[15] TUAN, H. D., KHA, H. H., NGUYEN, H. H., LUONG, V.J. Optimized training sequences for spatially correlated MIMOOFDM. IEEE Trans. on Wireless Communication, 2010, vol. 9, no. 9, p. 2768-2778. DOI: 10.1109/TWC.2010.070710.081601

[16] LUONG, V. D., TRAN, N. N., TUAN, H. D. Optimal training sequence design for MIMO-OFDM in spatially correlated fading. In Proceedings of the $2^{\text {nd }}$ International Conference on Signal Processing and Communication Systems. Australia (Gold Coast), Dec. 2008, p. 1-6. DOI: 10.1109/ICSPCS.2008.4813697

[17] GOLOVINS, E., VENTURA, N. Optimal training for the SMMIMO-OFDM systems with MMSE channel estimation. In Proceedings of the $6^{\text {th }}$ Annual Communication Networks and Services Research Conf. Halifax (Canada), 2008, p. 470-477. DOI:10.1109/CNSR.2008.57

[18] XIN GENG, HANYING HU, WEIJIA CUR, YANAN DUN. Optimal pilot design for MIMO OFDM channel estimation. In Proceedings of the International Conference on Signal Processing Systems, 2010, vol. 2, p. V2-404-V2-408.

[19] KAY, S. M. Fundamentals of Statistical Signal Processing: Estimation Theory. Prentice Hall, 1993.

[20] YUSHI SHEN, MARTINEZ, E. Channel estimation in OFDM systems. Freescale Semiconductor Application Note, 2006, Rev 0, 1/2006, p. 1-15.

[21] SAVAUX, V, LOUËT, Y, DJOKO-KOUAM, M., SKRZYPCZAK, A. Minimum mean-square-error expression of LMMSE channel estimation in SISO OFDM systems. IET Electronics Letters, 2013, vol. 49, no. 18, p. 1152-1154. DOI: 10.1049/el.2013.1993

[22] BIGUESH, M., GERSHMAN, A. B. Training based MIMO channel estimation: A study of estimator tradeoffs and optimal training signals. IEEE Transactions on Signal Processing, 2006, vol. 54, no. 3, p. 884-893. DOI: 10.1109/TSP.2005.863008

[23] 3GPP TS 36.101. User Equipment (UE) Radio Transmission and Reception.3rd Generation Partnership Project; Technical Specification Group Radio Access Network; Evolved Universal Terrestrial Radio Access (E-UTRA). 


\section{About the Authors ...}

Kaleeswaran RAJESWARI received A.M.I.E degree in Electronics and Communication Engineering from the Institute of Engineers (India) in 1996 and M.E from Madurai Kamaraj University, Madurai, India in 2003. She is currently doing $\mathrm{PhD}$ in Anna University, Chennai. She has a working experience as a lecturer for 12 years and as an assistant professor for 5 years. She is currently an Assistant Professor in the Electronics and Communication Engineering Department, Thiagarajar College of Engineering, Madurai. Her research interests include digital signal processing, statistical signal processing and MIMOOFDM wireless communication.
S. Jayaraman THIRUVENGADAM received the B.E. degree in Electronics and Communication Engineering from Thiagarajar College of Engineering, Madurai, India in 1991, the M.E degree in Applied Electronics from Anna University, Chennai, India, in 1994, and the $\mathrm{PhD}$ degree from Madurai Kamaraj University, Madurai, India in 2005. From Jan. to Dec. 2008, he was a Visiting Associate Professor with the Dept. of Electrical Engineering, Stanford University, CA under a Postdoctoral Fellowship, by the Dept. of Science and Technology, Government of India. $\mathrm{He}$ is currently a Professor with the Dept. of Electronics and Communication Engineering, Thiagarajar College of Engineering. His areas of research interest include statistical signal processing and MIMO wireless communication. 